\title{
Consistency theory in semiconservative spaces
}

\section{by}

A. K. SNYDER (Bethlehem, Pa.)

\begin{abstract}
Useful sufficient conditions are provided on a sequence space $\dot{V}$ such that every matrix summing $\nabla$ must be conull. This results in generalizations of Copping's conullity theorem and part of the classical consistency theory of matrices to a semiconservative setting. For certain sequence spaces $K_{0}$ and $V$ it is shown that $K_{0}^{\beta}$ is $\sigma\left(K_{0}^{\beta}, W_{E} \cap V\right)$ sequentially complete for all $\mathrm{FK}$ spaces $E$ containing $K_{0}$ whenever the multiplier algebra of $\nabla$ contains sufficiently many slowly oscillating sequences. Known completeness and consistency theorems for almost convergence follow as corollaries.
\end{abstract}

\$1. Introduction. A theorem of J. Copping states that every matrix which sums the bounded convergence domain of a conull matrix must be conull. This property of conullity is known to be equivalent to the Mazur-Orlicz Bounded Consistency Theorem. Furthermore, G. Bennett and $\mathrm{N}$. Kalton have observed that portions of the classical consistency theory of matrices can be reduced to problems involving the sequential completeness of $l^{1}$, the space of absolutely summable sequences, under appropriate weak topologies. The above work is placed in the context of FK spaces containing $c_{0}$, the space of null sequences.

We shall develop a consistency theory for semiconservative spaces which extends the above results. An essential feature of this extension is the provision of useful sufficient conditions on a sequence space $V$ such that every matrix summing $V$ must be conull. Copping's conullity theorem is thereby generalized to an appropriate semiconservative setting. For eertain sequence spaces $K_{0}$ and $V$ we show that $K_{0}^{\beta}$ is $\sigma\left(K_{0}^{\beta}, W_{E} \cap V\right)$ sequentially complete for all FK spaces $E$ containing $K_{0}$ whenever the multiplier algebra of $V$ contains sufficiently many slowly oscillating sequences.

As an application we observe that the multiplier algebra of $a c_{0}$ contains appropriate oscillating sequences, where $a c_{0}$ is the space of sequences which are almost convergent to zero. Known completeness and consistency theorems for almost convergence follow from this one essential property of $a c_{0}$. 
The writer wishes to thank Professors R. DeVos and A. Wilansky for helpful conversations during the preparation of this article. The important motivating influence of the consistency theorems for almost convergence of G. Bennett and N. Kalton is also acknowledged.

The following notation will be used:

$e, e^{n}$ are the sequences given by $e_{l_{k}}=1$ for all $k$ and $e_{k}^{n}=0$ for $k \neq n$, $e_{n}^{n}=1$

$\varphi$ is the linear span of $\left\{e^{n}: n=1,2, \ldots\right\}$;

$\omega$ is the space of all complex sequences;

$c_{0}=\{x \in \omega: \lim x=0\}$

$o=\{x \in \omega: \lim x$ exists $\}$;

$m=\left\{x \in \omega:\|x\|_{\infty}=\sup \left|x_{k}\right|<\infty\right\} ;$

$l^{p}=\left\{x \in \omega:\|x\|_{p}=\left(\sum_{k}^{k}\left|x_{k}\right|^{p}\right)^{1 / p}<\infty\right\}$ for $1 \leqslant p<\infty ;$

$b v=\left\{x \in \omega:\|x\|_{b v}=\lim \left|x_{k}\right|+\sum_{k}\left|x_{k}-x_{k+1}\right|<\infty\right\} ;$

$b s=\left\{x \in \omega:\|x\|_{b s}=\sup _{n}\left|\sum_{k=1}^{n} x_{k}\right|<\infty\right\} ;$

$c s=\left\{x \in \omega: \sum_{k} x_{k}\right.$ is convergent $\}$ with $\|x\|_{c s}=\|x\|_{b s}$.

Note for $x \in b s$ and $y \in \varphi$ that $\left|\sum_{k} x_{k} y_{k}\right| \leqslant\|x\|_{b s}\|y\|_{b v}$.

A sequence space is a linear subspace of $\omega$. A sequence space $E$ is an FK space if $E$ is a locally convex Fréchet space on which the coordinate functionals $x \rightarrow x_{n}$ are continuous for each $n$. An FK space whose topology is normable is a BK space. The spaces $c_{0}, c$, and $m$ are BK spaces under the norm of $m$. The spaces $l^{p}, b v, b s$, and $c s$ are BK spaces under the indicated norms.

We shall consider only sequence spaces containing $\varphi$.

For $x \in \omega$ let $P_{n} x=\sum_{k=1}^{n} x_{k} e^{k}$. If $E$ is an FK space, then

$$
W_{E}=\left\{x \in E: P_{n} x \rightarrow x \text { weakly in } E\right\} .
$$

The space $E$ is an AK space if $P_{n} x \rightarrow x$ for all $x \in E$.

Let $\langle E, F\rangle$ be paired linear spaces under a bilinear form $\langle\cdot, \cdot\rangle$. A sequence $\left\{x^{n}\right\}$ in $E$ is $\sigma(E, F)$ Cauchy if the map $y \rightarrow\left\{\left\langle x^{n}, y\right\rangle\right\}$ takes $F$ into $c$. An FK space $E$ is called semiconservative if $\left\{P_{n} e\right\}$ is $\sigma\left(E, Z^{\prime}\right)$ Cauchy, where $E^{\prime}$ is the space of continuous linear functionals on $E$. $E$ is called conull if $e \in W_{E}$, i.e. if $\left\{P_{n} e\right\}$ is $\sigma\left(E, E^{\prime}\right)$ convergent in $E$. Hence, comull spaces are semiconservative.

If $V$ and $W$ are sequence spaces, let $M(V, W)$ denote the sequence space of multipliers of $V$ into $W$. Thus, $u \in M(V, W)$ if and only if $u v$ $=\left\{u_{k} v_{k}\right\} \in W$ for all $v \in V$. For any $u \in \omega$ let $u V=\{u v: v \in \nabla\}$. If $E$ and $F$ are BK spaces, then $M(E, F)$ may be identified as a space of bounded maps from $E$ into $F$, and $M(E, F)$ is a BK space under the operator norm. Let $M(V)$ denote $M(V, V)$.

If $V$ is a sequence space, let $V^{\beta}=M(V, c s)$. If $V$ and $W$ are sequence spaces such that $V \subset W^{\beta}$, then the bilinear form $\langle v, w\rangle=\sum_{k} v_{k} w_{k}$ is defined on $V \times W$. We shall be concerned with the weak topology $\sigma(V, W)$ on $V$ relative to the pairing $\langle V, W\rangle$.

Finally, we shall consider matrix maps on sequence spaces. Let $A=\left(a_{n k}\right), n, k=1,2, \ldots$, be an infinite matrix of scalars. If . $x \in \omega$ and $\sum_{k} a_{n k} x_{k}$ converges for all $n$, let $A x$ be the sequence given by

$$
(A x)_{n}=\sum_{k} a_{n k} x_{k} .
$$

If $V$ is a sequence space, let $V_{A}=\{x \in \omega: A x \in V\}$. The space $e_{A}$ is the convergence domain of $A$. It is known that $c_{A}$ is an FK space. The matrix $A$ is called semiconservative if $c_{A}$ is semiconservative. The functional $\lim \in c_{A}^{\prime}$ is defined by $\lim _{A} x=\lim (A x)$. Whenever $e \in c_{\mathcal{A}}$ and $\left\{\lim _{\boldsymbol{A}} e^{k}\right\} \in \mathcal{A}$, we let

$$
\chi(A)=\lim _{n} \sum_{k} a_{n k}-\sum_{k} \lim _{n} a_{n k}=\underset{A}{\lim e}-\sum_{k} \lim _{A} e^{k} .
$$

Let $A$ be a semiconservative matrix with $e \in c_{A} . A$ is called conull if $\chi(A)=0$. It is known that $c_{A}$ is conull if and only if $A$ is conull. When dealing with the FK space $c_{\mathcal{A}}$ we shall abbreviate $W_{c_{A}}$ by $W_{\mathcal{A}}$. A matrix $A$ is row finite if the rows of $A$ are in $\varphi$.

The required properties of FK spaces and convergence domains may be found in [7] or [8]. See [5] for a discussion of semiconservative spaces.

\$2. Pseudoconull spaces. A sequence space $V$ will be called pseudoconull if every convergence domain containing $V$ is conull. Then every conull FK space is pseudoconull. The standard example of a pseudoconull space which is not conull is $m$. In this section we provide a useful sufficient condition for a sequence space to be pseudoconull.

Let $m$ and $n$ be positive integers, $m<n$. Then $[m, n]$ will denote the interval of positive integers $\{k: m \leqslant k \leqslant n\}$.

A sequence space $V$ will be said to have the (strong) oscillating sequence property if there exists a constant $M$ with the property that for each disjoint increasing sequence of intervals of positive integers there exists a subsequence $\left\{I_{k}\right\}, I_{k}=\left[m_{k}, n_{k}\right]$, and a sequence $\left\{v^{k}\right\} \subset \omega$ such that for each $k$, 
(i) $v_{i}^{k}=0$ for all $i \notin\left[n_{2 k-1}+1, m_{2 k+1}-1\right]$;

(ii) $v_{i}^{k}=1$ for all $i \in I_{2 l i}$;

(iii) $\left\|v^{k}\right\|_{b v} \leqslant M$; and

(iv) the pointwise $\operatorname{sum} \sum_{k} v^{k} \in V\left(\sum_{k} v^{\alpha_{k}} \in V\right.$ for every subsequence $\left\{v^{q_{k}}\right\}$ of $\left.\left\{v^{k}\right\}\right)$.

Of course, if $V$ has the (strong) oscillating sequence property, then so does $V \cap m$.

LEMMa 1. Let the matrix $A$ be row finite with $e \in c_{A}$ and $\left\{\lim e^{k}\right\} \in c s$. If $c_{A}$ has the oscillating sequence property, then $\chi(A)=0$.

Proof. Let $M$ be a constant given by the oscillating sequence property. Assume that $\chi(A) \neq 0$. Let $a$ be the sequence $\left\{\lim e^{k}\right\}$. Since os is an $\mathrm{AK}$ space, there exists an increasing sequence $\left\{l_{k}\right\}$ of positive integers such that

$$
\sum_{k}\left\|a-P_{l_{k-1}} a\right\|_{c s}<\frac{1}{3 M}|\chi(A)| .
$$

Ohoose sequences $\left\{p_{k}\right\},\left\{m_{k}\right\},\left\{n_{k}\right\}$ of positive integers as follows: Let $p_{1}=1, m_{1}=l_{1}$. Choose $n_{1}>m_{1}$ so that $a_{p_{1}, i}=0$ for $i \geqslant n_{1}$. Assume $p_{k}, m_{k}, n_{k}$ have been chosen. Ohoose $m_{k+1}>\max \left\{n_{k}, l_{k+1}\right\}$. Choose $p_{k+1}$ $>p_{k}$ so that

$$
\sum_{i=1}^{m_{k+1}}\left|a_{i}-a_{p_{k+1}, i}\right|<1 / k \text {. }
$$

Choose $n_{k+1}>m_{k+1}$ so that $a_{p_{k+1}, i}=0$ for $i \geqslant n_{k+1}$.

By hypothesis there exists a subsequence $\left\{\left[r_{k}, s_{k}\right]\right\}$ of the intervals $\left\{\left[m_{k}, n_{k}\right]\right\}$ and a sequence $u \in c_{A}$ so that

$$
\begin{gathered}
u_{i}=0 \quad \text { for } \quad i \leqslant r_{1} ; \\
u_{i}=0 \quad \text { for } \quad r_{2 k-1} \leqslant i \leqslant s_{2 k-1} ; \\
u_{i}=1 \quad \text { for } \quad r_{2 k} \leqslant i \leqslant s_{2 k} ; \\
\left\|\sum_{i=r_{k}}^{r_{k+1}-1} u_{i} e^{i}\right\|_{b v} \leqslant M .
\end{gathered}
$$

By deleting rows of $A$ we may assume that $A$ has the following form:

$$
\begin{gathered}
\sum_{i=1}^{r_{k}}\left|a_{i}-a_{k i}\right| \rightarrow 0 \quad \text { as } \quad k \rightarrow \infty ; \\
a_{k i}=0 \text { for } \quad i \geqslant s_{k} .
\end{gathered}
$$

Finally, we may assume that $a_{k i}=a_{i}$ for $1 \leqslant i \leqslant r_{k}$, since $u \in m$. We are adjusting $A$ by adding a matrix $B$ with $B u \in c$ and $\chi(B)=0$.
Note next that

But

$$
(A u)_{2 k}=\sum_{i} a_{2 k, i} u_{i}=\sum_{j=1}^{2 k-1}\left(\sum_{i=r_{j}}^{r_{j+1}-1} a_{i} u_{i}\right)+\sum_{i=r_{2 l}}^{8_{2 k}} a_{2 k, i} .
$$

$$
\begin{aligned}
\sum_{j=1}^{2 k-1}\left|\sum_{i=r_{j}}^{r_{j+1}-1} a_{i} u_{i}\right| & \leqslant \sum_{j=1}^{2 k-1}\left\|a-P_{l_{j-1}} a\right\|_{c s}\left\|\sum_{i=r_{j}}^{r_{j+1}-1} u_{i} e^{i}\right\|_{b v} \\
& \leqslant \frac{1}{3}|\chi(A)| .
\end{aligned}
$$

Also,

Therefore,

$$
\sum_{i=r_{2 k}}^{s_{2 k}} a_{2 k, i}=\sum_{i=1}^{\infty} a_{2 k, i}-\sum_{i=1}^{r_{2 k}-1} a_{i} \rightarrow \chi(A) .
$$

Similarly,

$$
\limsup \left|(A u)_{2 k}-\chi(A)\right| \leqslant \frac{1}{3}|\chi(A)| \text {. }
$$

As above,

$$
(A u)_{2 k+1}=\sum_{i} a_{2 k+1, i} u_{i}=\sum_{i=1}^{r_{2 k+1}-1} a_{i} u_{i}=\sum_{j=1}^{2 k}\left(\sum_{i=r_{j}}^{r_{j+1}-1} a_{i} u_{i}\right) .
$$

$$
\left|(A u)_{2 k+1}\right|<\frac{1}{3}|\chi(A)| \text {. }
$$

The contradiction $A u \notin e$ follows easily.

Lenara 2. Let $K_{0}$ be a semiconservative $\mathrm{FK}$ space, and let $V$ be a sequence space satisfying $K_{0} \subset \nabla \subset M\left(K_{0}^{\beta}\right)$. Then $K_{0}^{\beta}=\nabla^{\beta}$.

Proof. The inclusion $V^{\beta} \subset K_{0}^{\beta}$ is obvious.

Let $u \in K_{0}^{\beta}$. If $v \in V$, then $u v \in K_{0}^{\beta}$, since $\nabla \subset M\left(K_{0}^{\beta}\right)$. Also, $K_{0}^{\beta} \subset c s$, since $K_{0}^{\beta}$ may be identified as a subspace of the dual of the semiconservative space $K_{0}$. It follows that $u v \in c s$, so $u \in V^{\beta}$.

Recall that if $K_{0}$ is a BK space, then $K_{0}^{\beta}$ is a natural BK space under the norm of $M\left(K_{0}, o s\right)$.

THEOREM 1. Let $K_{0}$ be a semiconservative $\mathrm{BK}$ space such that $\varphi$ is dense in $K_{0}^{\beta}$ and $K_{0} \subset M\left(K_{0}^{\beta}\right)$. Let $V$ be a sequence space such that $e \in V$ and $K_{0} \subset V \subset M\left(K_{0}^{\beta}\right)$. If $V$ has the oscillating sequence property, then $V$ is pseudoconull.

Proof. Assume that $V \subset c_{A}$. Let $a^{n}$ be the $n^{\text {th }}$ row of the matrix $A$. Since $\varphi$ is dense in $K_{0}^{\beta}$, there exists $b^{n} \in \varphi$ for each $n$ such that $a^{n}-b^{n} \rightarrow 0$ in $K_{0}^{\beta}$. Let $B$ be the matrix whose $n^{\text {th }}$ row is $b^{n}$.

Note next that $V \subset c_{B}$, for suppose $v \in V$. By Lemma $2, K_{0}^{\beta}=M\left(K_{0}^{\beta}\right)^{\beta}$. Furthermore, $a^{n}-b^{n} \rightarrow 0$ in $K_{0}^{\beta}=M\left(K_{0}^{\beta}\right)^{\beta}=M\left(M\left(K_{0}^{\beta}\right), c s\right)$ and $v \in M\left(K_{0}^{\beta}\right)$. It follows that $\left(a^{n}-b^{n}\right) v \rightarrow 0$ in $c s$, so

$$
\sum_{i} a_{i}^{n} v_{i}-\sum_{i} b_{i}^{n} v_{i} \rightarrow 0
$$

Thus, $V \subset c_{B}$ since $V \subset c_{A}$. 
But $B$ is row finite. Also, $e \in V \subset c_{B}$. Finally, $K_{0}$ is semiconservative and $K_{0} \subset V \subset c_{B}$, so $c_{B}$ must be semiconservative. Hence $\left\{\lim _{B} e^{k}\right\} \in c s$. By Theorem $1, \chi(B)=0$, so $B$ is conull

Now $A-B$ maps $\varphi$ and $e$ into $c_{0}$ according to (1), so $\chi(A-B)=0$. Since $B$ and $A-B$ are conull, it follows that $A$ is conull.

We next provide a large class of sequence spaces with the oscillating sequence property.

LiEMMa 3. Let te be a semiconservative FK space, and let $\left\{p_{n}\right\}$ be an increasing sequence of positive integers. For $x \in E$ let $x^{n}=\sum_{k=p_{n}+1}^{p_{n+1}} x_{l c} e^{k}$. If the series $\sum_{n} x^{n}$ converges weakly in $E$ and $\left\{x^{n}\right\}$ is bounded in bv, then $x \in W_{x}$.

Proof. If $f \in E^{\prime}$, then $\left\{f\left(e^{k}\right)\right\} \in c s$, since $E$ is semiconservative. Also,

$$
\left\|\sum_{l=p_{n}+1}^{p_{n+1}} f\left(e^{l k}\right) e^{k}\right\|_{c s} \rightarrow 0
$$

since os is an AK space.

For any integer $m$ suppose $p_{n}<m \leqslant p_{n+1}$. Now

$$
f\left(\sum_{k=n+1}^{\infty} x^{k}\right) \rightarrow 0 \quad \text { as } \quad n \rightarrow \infty
$$

and

$$
\left|\sum_{k=m+1}^{p_{n+1}} x_{k} f\left(e^{k}\right)\right| \leqslant\left\|\sum_{k=p_{n}+1}^{p_{n+1}} f\left(e^{k}\right) e^{k}\right\|_{c s}\left\|\sum_{l=m}^{p_{n+1}+1} x_{l c} e^{k}\right\|_{b v} \leqslant 2\left\|\sum_{k=p_{n}+1}^{p_{n+1}} f\left(e^{k}\right) e^{k}\right\|_{c s}\left\|x^{n}\right\|_{b v}
$$

Thus, since

$$
\rightarrow 0 \quad \text { as } \quad n \rightarrow \infty \text {. }
$$

it follows that

$$
f(x)=\sum_{k=1}^{m} x_{k} f\left(e^{k}\right)+\sum_{k=m+1}^{p_{n+1}} x_{k} f\left(e^{l_{k}}\right)+f\left(\sum_{k=n+1}^{\infty} x^{k}\right)
$$

$$
f(x)=\sum_{k=1}^{\infty} x_{k} f\left(e^{k}\right)
$$

Therefore, $x \in W_{E}$.

LEMMA 4. If $E$ is a conull FK space, then $W_{E}$ has the strong oscillating sequence property.

Proof. By hypothesis, $e-P_{n} e \rightarrow 0$ weakly in $E$, so appropriate convex combinations of the sequence $\left\{e-P_{n} e\right\}$ will converge to 0 in $E$. Hence, there exists $\left\{x^{k}\right\} \subset E$ such that for each $k$

(a) $x_{i}^{k}=1$ for $i$ large;

(b) $0 \leqslant x_{i}^{k} \leqslant 1$ for all $i$ (c) $x_{i}^{k}$ is nondecreasing;

(d) $! x^{k} !<2^{-k}$

where ! $\cdot$ ! is a paranorm providing the topology of $E$. Now $x^{k} \rightarrow 0$ pointwise. Thus, we may assume without loss of generality that $x_{i}^{k}=0$ for $1 \leqslant i \leqslant k$.

Let $p_{1}=1$. Choose an interval $I_{1}=\left[m_{1}, n_{1}\right]$ from the given sequence of intervals such that $x_{i}^{p_{1}}=1$ for all $i \geqslant m_{1}$. Assume that $p_{k}$ and $I_{k}=\left[m_{k}\right.$, $n_{k}$ ] have been chosen. Choose $p_{k+1}>\max \left\{p_{k}, n_{k}\right\}$. Then choose $I_{k+1}$ $=\left[m_{k+1}, n_{k+1}\right]$ from the given sequence of intervals with $m_{k+1}>n_{k}$ so that $x_{i}^{p} k+1=1$ for all $i \geqslant m_{k+1}$.

By this process one obtains a sequence $\left\{u^{k}\right\} \subset E$ and a subsequence of intervals $I_{k}=\left[m_{k}, n_{k}\right]$ such that for each $k$

(a) $u_{i}^{k}=1$ for $i \geqslant m_{k}$ and $u_{i}^{k}=0$ for $i \leqslant n_{k-1}$;

(b) $0 \leqslant u_{i}^{k} \leqslant 1$ for all $i$;

(c) $u_{i}^{k}$ is nondecreasing;

(d) $! u^{k} !<2^{-k}$.

Let $v^{k}=u^{2 k}-u^{2 k+1}$ for each $k$. The conditions (i) through (iii) of the strong oscillating sequence property are satisfied for the constant $M=2$.

Let $\left\{q_{k}\right\}$ be an increasing sequence of positive integers. The series $\sum v^{q_{k}}$ converges absolutely in $E$, hence weakly. By Lemma $3, \sum_{k} v^{q_{k}} \in W_{E}$, so condition (iv) is satisfied.

Let $V$ be a sequence space. Assume that for each increasing sequence $\left\{p_{n}\right\}$ of positive integers and for each sequence $\left\{x^{n}\right\} \subset \omega$ satisfying $x_{i}^{n}=0$ for $i \notin\left[p_{n}, p_{n+1}\right]$ and $\left\{x^{n}\right\}$ bounded in $b v$, there exists a subsequence $\left\{x^{q_{n}}\right\}$ such that the pointwise sum $\sum_{n} x^{q_{n}} \in V$. Then $V$ will be said to have the gliding humps property.

LEMMA 5. Let $\nabla$ and $W$ be sequence spaces. If $V$ has the strong oscitlating sequence property and $W$ has the gliding humps property, then $V \cap W$ has the oscillating sequence property.

Proof. Given a disjoint increasing sequence of intervals of positive integers, choose a subsequence $\left\{I_{k_{t}}\right\}$ and a sequence $\left\{v^{k}\right\} \subset \varphi$ satisfying conditions (i) through (iv) of the definition for the strong oscillating sequence property of $\nabla$. Since $W$ has the gliding humps property, there is a subsequence $\left\{v^{a_{k}}\right\}$ so that $\sum_{k} v^{q_{k}} \in W$. But $\sum_{k} v^{a_{k}} \in V$ as well.

THEOREM 2. Let $K_{0}$ be a semiconservative BK, AK space such that $p$ is dense in $K_{0}^{\beta}$ and $K_{0} \subset M\left(K_{0}\right)$. Let $V$ be a sequence space such that $e \in V$ and $K_{0} \subset V \subset M\left(K_{0}^{\beta}\right)$. If $E$ is a conull FK space, $K_{0} \subset E$, and if $V$ has the gliding humps property, then $W_{E} \cap V$ is pseudoconull.

Proof. Note that $K_{0} \subset W_{E}$ since $K_{0}$ is an AK space. By Lemma 4 and Lemma $5, W_{E} \cap V$ has the oscillating sequence property. Therefore, Theorem 1 applies. 
Theorem 2 generalizes Copping's Theorem [3], Theorem 3, to a semiconservative setting. If $K_{0}=c_{0}$, then $M\left(K_{0}^{\beta}\right)=m$. Clearly, $V=m$ has the gliding humps property.

Other proofs of Copping's Theorem have been given by Wilansky in [6] and by Bennett and Kalton in [1].

Using matrices in the setting of spaces containing $c_{0}$, one can provide spaces with the oscillating sequence property without resorting to conullity.

THEOREM 3. Let $V$ be a sequence space satisfying $c_{0} \subset V \subset m$, and let $A$ be a matrix with nuth columns such that $c \subset V_{A}$. If $M(V)$ has the gliding humps property, then $\nabla_{A}$ has the oscillating sequence property. Hence $V_{A} \cap m$ is pseudoconull.

Pro of. Since $A(c) \subset V \subset m$, it is known that $\|A\|=\sup _{n} \sum_{k}\left|a_{n k}\right|<\infty$. Without loss of generality we may assume that the rows and columns of $A$ are in $\varphi$.

Choose increasing sequences $\left\{m_{n}\right\},\left\{k_{n}\right\}$ of positive integers as follows: Choose $m_{1}$ so that $a_{1 i}=0$ for all $i \geqslant m_{1}$. Choose $k_{1}$ so that $a_{p i}=0$ for $p \geqslant k_{1}$ and $1 \leqslant i \leqslant m_{1}$. Assume $m_{n}$ and $k_{n}$ have been chosen. Choose $m_{n+1}>m_{n}$ so that $a_{p i}=0$ for $i \geqslant m_{n+1}$ and $1 \leqslant p \leqslant k_{n}$. Then choose $k_{n+1}>k_{n}$ so that $a_{p i}=0$ for $p \geqslant k_{n+1}$ and $1 \leqslant i \leqslant m_{n+1}$.

If $M(V)$ has the gliding humps property, the following construction is possible: Given a disjoint increasing sequence of intervals of positive integers, there exists a subsequence $\left\{I_{n}\right\}$ and a scalar sequence $\left\{t_{i}\right\}$ satisfying

(i) $0 \leqslant t_{i} \leqslant 1$ for all $i$;

(ii) $t_{i+1}-t_{i} \rightarrow 0$ as $i \rightarrow \infty$;

(iii) Let $u$ be given by $u_{j}=t_{i}$ for $m_{2 i-2} \leqslant j<m_{2 i}$. Then for each $n, u_{j}=0$ on $I_{2 n-1}, u_{j}=1$ on $I_{2 n}, u_{j}$ is nondecreasing between $I_{2 n-1}, I_{2 n}$ and $u_{j}$ is nonincreasing between $I_{2 n}, I_{2 n+1}$.

(iv) Let $v$ be given by $v_{j}=t_{i}$ for $k_{2 i-2} \leqslant j<k_{2 i}$. Then $v \in M(V)$.

Observe next that $v(A e)-A u \in c_{0}$, for suppose $n$ is a given positive integer. Choose $i$ so that $k_{2 i-1} \leqslant n<k_{2 i+1}$. If $k_{2 i-1} \leqslant n<k_{2 i}$, then

$$
\begin{aligned}
v_{n}(A e)_{n}-(A u)_{n} & =v_{n} \sum_{j} a_{n j}-\sum_{j} a_{n j} u_{j} \\
& =t_{i} \sum_{j=m_{2 i-1}}^{m_{2 i+1}} a_{n j}-\left(t_{i} \cdot \sum_{j=m_{2 i-1}}^{m_{2 i}-1} a_{n j}+t_{i+1} \sum_{j=m_{2 i}}^{m_{2 i+1}} a_{n j}\right) \\
& =\left(t_{i}-t_{i+1}\right) \sum_{j=m_{2 i}}^{m_{2 i+1}} a_{n j} .
\end{aligned}
$$

Similarly, if $k_{2 i} \leqslant n<k_{2 i+1}$, then

$$
v_{n}(A e)_{n}=t_{i+1} \sum_{j=m_{2 i}}^{m_{2 i+2}} a_{n j}=(A u)_{n} \text {. }
$$

Now $A e \in V$ so $v(A e) \in V$, since $v \in M(V)$. Therefore, $A u \in V$. We have shown that $V_{A}$ has the oscillating sequence property. According to Theorem 1, $\nabla_{A} \cap m$ must be pseudoconull.

§ 3. A semiconservative consistency theory. In this section we develop for appropriate semiconservative spaces a consistency theory based on the psieudoconull property.

Let $u \in \omega$ with $u_{i} \neq 0$ for all $i$, let $E$ be an FK space, and let $A=\left(a_{n_{k}}\right)$ be a matrix. Let $\frac{1}{u}$ denote the sequence $\left\{\frac{1}{u_{n}}\right\}$. Then $\frac{1}{u} W_{E}=W_{\frac{1}{u} E}$, so $u \in W_{E}$ if and only if $\frac{1}{u} E$ is conull. Also, note that for any sequence space $\nabla, \frac{1}{u} V_{A}=V_{A u}$ where $A u$ is the matrix $\left(a_{n k} u_{k}\right)$. In what follows it will be clear from the context whether the symbol $A u$ represents a matrix or a sequence.

LEMMA 6. Let $K_{0}$ be an FK, AK space such that $K_{0} \subset M\left(K_{0}\right)$. Let $V$ be a sequence space such that $K_{0} \stackrel{\subset}{\odot} \subset M\left(K_{0}\right)$. Assume that $W_{E} \cap M(\nabla)$ is pseudoconull for all conull FK spaces $E \supset K_{0}$. Then for all FK spaces $E \supset K_{0}$, $W_{E} \cap V \subset W_{A}$ whenever $W_{E} \cap V \subset c_{A}$.

Proof. Assume that $W_{E} \cap V \subset c_{A}$ and $u \in W_{E} \cap V$. We may assume without loss of generality that $u_{i} \neq 0$ for all $i$, because there exists $w \in \boldsymbol{K}_{0}$ such that $u_{i}+w_{i} \neq \mathbf{0}$ for all $i$. Then $w \in W_{A}$ since $K_{0}$ is an AK space.

Now $\frac{1}{u} W_{E} \cap \frac{1}{u} V \subset \frac{1}{u} c_{\mathcal{A}}$, and $\frac{1}{u} E$ is conull. Also, $K_{0} \subset \frac{1}{u} E$ since $V \subset M\left(K_{0}\right)$ and $K_{0} \subset E$. Finally, $M(V) \subset \frac{1}{u} V$ since $u \in V$. Therefore, $W_{\frac{1}{u} E} \cap M(V) \subset c_{A u}$. By hypothesis, $c_{A u}=\frac{1}{u} c_{A}$ is conull, so
$u \in W_{A}$.

Note that if a matrix $A$ is semiconservative and $t \in l^{1}$, then $\sum_{k} \sum_{i} t_{k} a_{k i}$ $=\sum_{i} \sum_{k} t_{k} a_{k i}$. See the proof of Theorem 6 of [5].

LEMMA 7. Let $K_{0}$ be a semiconservative FK space such that $K_{0} \subset M\left(K_{0}\right)$. Let $V$ be a sequence space with $K_{0} \subset V \subset M\left(K_{0}\right)$. Then $V \subset W_{A}$ whenever $V \subset c_{\mathcal{A}}$ if and only if $K_{0}^{\beta}$ is $\sigma\left(\boldsymbol{K}_{0}^{\beta}, V\right)$ sequentially complete.

Proof. Assume that $V \subset W_{A}$ whenever $V \subset c_{A}$. Now $M\left(K_{0}\right)$ $\subset M\left(K_{0}^{\beta}\right)$ so by Lemma $2, K_{0}^{\beta}=\nabla^{\beta}$ and the duality exists. Let $\left\{a^{n}\right\}$ be a $\sigma\left(K_{0}^{\beta}, V\right)$ Cauchy sequence in $K_{0}^{\beta}$. Let $A$ be the matrix whose $n^{\text {th }}$ row is $a^{n}$. 
Then $V \subset c_{A}$ so $\nabla \subset W_{A}$. Let $a=\left\{\lim e^{i}\right\}$. If $v \in V$, then $P_{n} v \rightarrow v$ weakly in $c_{A}$, so $\lim P_{n} v \rightarrow \lim v$, i.e.

$$
\sum_{i} a_{i} v_{i}=\lim _{n} \sum_{i} a_{n i} v_{i}
$$

Thus, $a^{n} \rightarrow a$ in $\sigma\left(K_{0}^{\beta}, \nabla\right)$.

Conversely, suppose $V \subset c_{A}$. Let $a^{n}$ be the $n^{\text {th }}$ row of $A$. Then $\left\{a^{n}\right\}$ is $\sigma\left(K_{0}^{\beta}, V\right)$ Cauchy, so $a^{n} \rightarrow a$ in $\sigma\left(K_{0}^{\beta}, V\right)$. Let $f \in c_{A}^{\prime}$. Now if $x \in K_{0}$ and $v \in V$, then $v x \in K_{0}$. Hence, $K_{0} \subset c_{A v}$, so the matrix $A v$ is semiconservative. Thus,

$$
\sum_{i} \sum_{i} t_{i} a_{i k} v_{k}=\sum_{i} \sum_{k} t_{i} a_{i k} v_{k} .
$$

The usual representation for $c_{\mathcal{A}}^{\prime}$ yields

$$
f(v)=\underset{\mathcal{A}}{\alpha \lim _{i}} x+\sum_{k} t_{k}(A v)_{k}+\sum_{i} b_{i} v_{i}, \quad v \in V
$$

where $\alpha$ is a constant, $t \in l^{1}$ and $b \in c_{A}^{\beta}$. a But then

$$
f(v)=\alpha \sum_{i} a_{i} v_{i}+\sum_{i} v_{i}\left(\sum_{k} t_{k} a_{k i}\right)+\sum_{i} b_{i} v_{i}=\sum_{i} v_{i} f\left(e^{i}\right), \quad v \in V
$$

so $V \subset W_{A}$.

LEMMA 8. If $K_{0}$ is a semiconservative FK space and $V$ is a sequence space with $K_{0} \subset V \subset M\left(K_{0}\right)$, then $K_{0} \subset M(V) \subset M\left(K_{0}^{\beta}\right)$.

Proof. Let $x \in K_{0}, v \in V$. Then $v \in M\left(K_{0}\right)$, so $v x \in K_{0} \subset V$. Therefore, $K_{0} \subset M(V)$.

To obtain the second inclusion, observe that $M(\nabla) \subset M\left(V^{\beta}\right)$. By Lemma $2, V^{\beta}=K_{0}^{\beta}$, so $M(\nabla) \subset M\left(K_{0}^{\beta}\right)$.

THEOREM 4. Let $K_{0}$ be a semiconservative $\mathrm{BK}$, AK space such that $\varphi$ is dense in $K_{0}^{\beta}$ and $K_{0} \subset M\left(K_{0}\right)$. Consider the following conditions on a sequence space $V$ satisfying $K_{0} \subset V \subset M\left(K_{0}\right)$.

(i) $M(V)$ has the gliding humps property;

(ii) For all conull spaces $E \supset K_{0}, W_{E} \cap M(V)$ is pseudoconull;

(iii) For all FK spaces $E \supset K_{0}, W_{E} \cap V \subset W_{A}$ whenever $W_{E} \cap V \subset c_{A}$;

(iv) For all FK spaces $E \supset K_{0}, K_{0}^{\beta}$ is $\sigma\left(K_{0}^{\beta}, W_{E} \cap \nabla\right)$ sequentially complete.

Then (i) implies (ii), (ii) implies (iii), and (iii) is equivalent to (iv). Also, (ii) is equivalent to (iii) if $\nabla=M(V)$.

Proof. Let $M(V)$ have the gliding humps property. Assume that $E$ is conull, $\boldsymbol{K}_{0} \subset E$. According to Lemma $8, K_{0} \subset M(V) \subset M\left(\boldsymbol{K}_{0}^{\beta}\right)$. By Theorem 2, $W_{E} \cap M(V)$ is pseudoconull, so (i) implies (ii).
Lemma 6 asserts that (ii) implies (iii) and Lemma 7 yields the equivalence of (iii) and (iv).

Finally, assume $V=M(V)$. If $E$ is conull, $K_{0} \subset E$, and $W_{E} \cap M(V)$ $\subset c_{A}$, then $e \in W_{A}$ by (iii). Therefore, $A$ is conull, so (iii) implies (ii).

The relatively strong conditions imposed on the base space $K_{0}$ in Theorem 4 can be satisfied for $K_{0} \neq \mathrm{c}_{0}$. An elementary computation shows that

$$
K_{0}=\left\{x \in c_{0}:\left\{x_{n+1}-x_{n}\right\} \in l^{p}\right\}, \quad 1<p<\infty
$$

provides such an example. It should be noted however that if $K_{0}$ is semiconservative, $K_{0} \subset M\left(K_{0}\right)$, and $\left\{e^{n}\right\}$ is an unconditional basis for $K_{0}$, then $K_{0}=c_{0}$. Also note that if $b v \cap c_{0} \subset K_{0}$ (for instance, $K_{0}$ semiconservative) and $K_{0} \subset M\left(K_{0}\right)$, then $K_{0}$ is an AK space if and only if $\varphi$ is dense in $K_{0}$.

It should be noted also that the techniques of [1] may be applied in Theorem 4 (iii) to replace the assumption that $W_{E} \cap V \subset c_{A}$ by $W_{E} \cap V$ $\subset F^{\prime}$ where $F^{\prime}$ is a separable $F K$ space.

The following result is a semiconservative analog to Theorem 11 of [2]. The proof is omitted, being a more or less routine semiconservative extension of the proof of Bennett and Kalton.

THEOREM 5. Let $K_{0}$ be a semiconservative AK space satisfying $K_{0}$ $\subset M\left(K_{0}\right)$, let $V$ be a sequence space with $K_{0} \subset V \subset M\left(K_{0}\right)$, and let $A$ be a matrix with $K_{0} \subset c_{A}$. Suppose that $K_{0}^{\beta}$ is $\sigma\left(K_{0}^{\beta}, W_{A} \cap \nabla\right)$ sequentially complete. If $c_{A} \cap V \subset c_{B}$, then there is a constant a such that

$$
\lim _{B} x-\sum_{k} x_{k} \lim _{B} e^{k}=\alpha\left(\lim _{A} x-\sum_{k} x_{k} \lim _{A} e^{k}\right)
$$

for all $x \in c_{A} \cap V$.

TheOREM 6. Let $V$ be a sequence space satisfying $c_{0} \subset V \subset m$, and let $A$ be a matrix mapping $c_{0}$ into $c_{0}$. If $M(V)$ has the gliding humps property, then $l^{1}$ is $\sigma\left(l^{1}, V_{A} \cap m\right)$ sequentially complete.

Proof. Assume that $V_{A} \cap m \subset c_{B}$. Now $c_{0} \subset V_{A} \cap m$, so $c_{0} \subset W_{B}$ since $c_{0}$ is an AK space. Let $u \in V_{A} \cap m$. As before, we may assume that $u_{i} \neq 0$ for all $i$. Then

$$
\frac{1}{u} V_{A} \cap \frac{1}{u} m \subset \frac{1}{u} c_{B}
$$

$$
V_{\perp u} \cap m \subset c_{B u} .
$$

According to Theorem $3, V_{A u} \cap m$ is pseudoconull, so $c_{B u}$ is conull. Therefore, $u \in W_{B}$.

We have shown that $V_{A} \cap m \subset W_{B}$ whenever $V_{A} \cap m \subset c_{B}$. Since $c_{0}^{\beta}=l^{1}$, Lemma 7 implies that $l^{1}$ is $\sigma\left(l^{1}, V_{\mathcal{A}} \cap m\right)$ sequentially complete. 
\$4. The consistency theory for almost convergence. Let ae be the BK space of almost convergent sequences, and let $a c_{0}$ be the codimension one subspace of sequences which are almost convergent to zero. A nice discussion of almost convergence may be found in [2] along with proofs of the following known characterizations of the space $a c_{0}$.

LEMMA 9. (i) $a c_{0}$ is the closure in $m$ of $b s$;

(ii) $x \in a c_{0}$ if and only if

$$
\lim _{p} \frac{1}{p} \sum_{k=n}^{n+p-1} x_{k}=0
$$

uniformly in $n$

Lemma 9 (ii) is the original characterization of Lorentz in [4]

In this section we show that $M\left(a c_{0}\right)$ has the gliding humps property. This observation identifies the essential property of $a c_{0}$ on which the consistency and completeness theorems for almost convergence of $G$. Bennett and N. Kalton depend. See [2], Theorems 6 and 8 .

If $S$ is a set, let $|S|$ denote the cardinality of $S$. For positive integers $n$ and $p$ let $J_{n p}$ be the interval $[n, n+p-1]$ of positive integers.

LEMMA 10. Every increasing sequence of intervals of positive integers has a subsequence $\left\{I_{m}\right\}$ such that

$$
\frac{1}{p} \sup _{n}\left|\left\{m: I_{m} \cap J_{n p} \neq \varphi\right\}\right| \rightarrow 0
$$

as $p \rightarrow \infty$.

Proof. Choose a subsequence $\left\{I_{m}\right\}, I_{m}=\left[i_{m}, j_{m}\right]$, of the given sequence of intervals such that

for all $m$.

$$
i_{m+1}-j_{m} \geqslant m
$$

For a given interval $J_{n p}$ suppose $I_{M}, I_{M+1}, \ldots, I_{M+k-1}$ are the members of $\left\{I_{m}\right\}$ which meet $J_{n p}$. Then

$$
p \geqslant \sum_{j=M+1}^{M+k-1} j \geqslant \frac{1}{2}(k-1) k .
$$

Hence, $(k-1)^{2} \leqslant(k-1) k \leqslant 2 p$, so $k \leqslant 1+(2 p)^{1 / 2}$. It follows that

$$
\frac{1}{p} \sup _{n}\left|\left\{m: I_{m} \cap J_{n p} \neq p\right\}\right| \leqslant \frac{1}{p}\left(1+(2 p)^{1 / 2}\right) \rightarrow 0
$$

as $p \rightarrow \infty$.

THEOREM 7. $M\left(a c_{0}\right)$ has the gliding humps property.

Proof. Let $\left\{p_{n}\right\}$ be an increasing sequence of positive integers. Using Lemma 10 let $\left\{I_{n}\right\}$ be a disjoint subsequence of $\left\{\left[p_{n}, p_{n+1}\right]\right\}$ satis- fying $\frac{1}{p} \sup _{n}\left|\left\{m: I_{m} \cap J_{n p} \neq p\right\}\right| \rightarrow 0$ as $p \rightarrow \infty$. Let $\left\{u^{n}\right\}$ be a sequence in bv such that $\left\|u^{n}\right\|_{b v} \leqslant M$ for all $n$ and $u_{i}^{n}=0$ for all $i \notin I_{n}$. Let $u$ be the pointwise sum $\sum u^{n}$.

Let $x \in b s$ be arbitrary. Now

$$
\begin{aligned}
\frac{1}{p}\left|\sum_{i=n}^{n+p-1} u_{i} x_{i}\right| \leqslant \frac{1}{p} \sum_{k=1}^{\infty}\left|\sum_{i \in I_{k} \cap J_{n p}} u_{i}^{k} x_{i}\right| & \leqslant \frac{1}{p} \sum_{I_{k} \cap J_{n p} \neq \varphi}\left\|u^{k}\right\|_{b v}\|x\|_{b s} \\
& \leqslant \frac{1}{p}\left|\left\{k: I_{k} \cap J_{n p} \neq \varphi\right\}\right| M\|x\|_{b s} .
\end{aligned}
$$

Thus, $\frac{1}{p} \sum_{i=n}^{n+p-1} u_{i} x_{i} \rightarrow 0$ uniformly in $n$ as $p \rightarrow \infty$, so $u x \in a c_{0}$. We have shown that $u \in M\left(b s, a c_{0}\right)$.

Finally, let $x \in a c_{0}$ be arbitrary. By Lemma 9 (i), choose $x^{n} \in b s$ so that $x^{n} \rightarrow x$ in $m$. Then $u x^{n} \in a c_{0}$ and $u x^{n} \rightarrow u x$ in $m$. Therefore, $u \in M\left(a c_{0}\right)$.

Taking $K_{0}=c_{0}$ and $V=a c_{0}$ in Theorems 4 and 6 , we obtain.

CoRoLLARY (Bennett-Kalton [2]). If $E$ is an FK space containing $c_{0}$ and $A$ is a matrix mapping $c_{0}$ into $c_{0}$, then $l^{1}$ is sequentially complete under the topologies $\sigma\left(l^{1}, W_{E} \cap a c_{0}\right)$ and $\sigma\left(l^{1},\left(a c_{0}\right)_{A} \cap m\right)$.

\section{References}

[1] G. Bennett and N. Kalton, FK epaces containing $c_{0}$, Duke Math. J. 39 (1972), $561-582$.

[2] - Consistency theorems for almost convergence, Trans. Amer. Math. Soc. 198 (1974), 23-43.

[3] J. Copping, Inclusion theorems for conservative summation methods, Nederl. Akad. Wetensch. Proc. Ser. A 61 (1958), 485-499.

[4] G. G. Lorentz, A contribution to the theory of divergent sequences, Acta Math. 80 (1948), 167-190.

[5] A. K. Snyder and A. Wilansky, Inolusion theorems and semiconservative $\mathrm{FK}$ spaces, Rocky Mountain J. Math. 2 (1972), 595-603.

[6] A. Wilansky, Topological divisors of zero and Tauberian theorems, Trans. Amcr. Math. Soc. 113 (1964), 240-251.

[7] A. Wilansky, Functional analysis, Ginn, New York 1964.

[8] K. Zeller and W. Beekman, Theorie der Limitierungsverfahren, Zweite, crweiterte und verbesserte Auflage, Ergebnisse der Math. und ihrer Grenzgebicte, Band 15, Springer-Verlag, Berlin and New York 1970. 